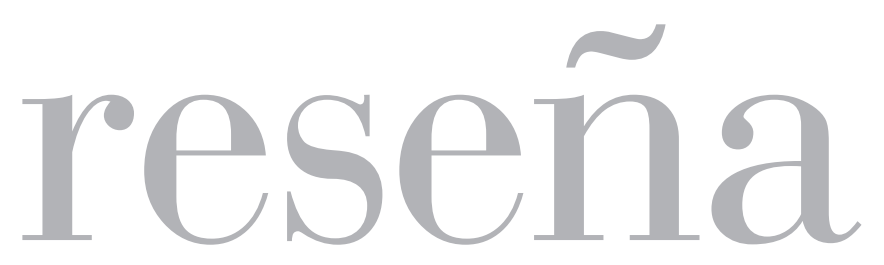





\section{La antropologia del estado desde Akhil Gupta: a propósito de Red Tape. Burocracia, violencia estructural $y$ pobreza en India.}

\section{Red Tape: Bureaucracy, Structural Violence, and Poverty in India. Akhil Gupta. 2012. Durham: Duke University Press. 368 pp.}

Julio Arias Vanegas ${ }^{1}$

Instituto Pensar, Universidad Javeriana, Bogotá ${ }^{2}$

julioariasvanegas@gmail.com

\footnotetext{
Investigador asociado

2 Candidato al doctorado en antropología, CUNY. Antropólogo y Magíster en antropologia, Universidad de los Andes, Bogotá
} 
Una mujer india de mediana edad sentada en su despacho frente a una mesa grande y desgastada donde posan sus lentes, su boligrafo y unos legajos, delante de armarios coronados por pilas y pilas de documentos envejecidos, es una de las imágenes del proyecto "Bureaucratics" del fotógrafo holandés Jan Banning. Este proyecto buscó, complicando la visión weberiana de la burocracia, plasmar la diversidad de los funcionarios estatales alrededor del mundo y destacar cómo manejan la información, algo muchas veces materializado en caóticos arrumes de carpetas y archivadores. La imagen de esta mujer india contratada por 'razones humanitarias' después de la muerte de su esposo en la misma oficina donde él trabajaba, protagoniza la portada del último libro del profesor de antropología Akhil Gupta: Red Tape (2012). Aunque es un detalle que Gupta no revela, la imagen dialoga con el título. Red tape es una expresión anglosajona para referir la excesiva rigidez y el sin fin del papeleo y de los procedimientos burocráti$\cos ^{3}$. De entrada el libro enfatiza en la materialidad del estado y pone sobre la mesa la relación entre estado, violencia y pobreza extrema. Precisamente, Red Tape empieza y termina con unas preguntas morales y políticas que pocos antropólogos llegan a hacerse: ¿por qué un estado como el indio "cuyo objetivo proclamado ha sido fomentar el desarrollo, no ha podido ayudar al abrumador número de personas que viven en la extrema pobreza?” (Gupta, 2012, p. 4) Y, “¿por qué sus muertes no generan un escándalo que deslegitime el régimen dominante?” (Gupta, 2012, p. 278).

Red Tape es una extensa reflexión alimentada por desespero y rabia, tal como Gupta señalaria después (Gupta, Nugent y Sreenath, 2015, p. 588), sobre cómo la violencia estructural ha sido constitutiva de la producción del estado en India en medio de políticas de desarrollo, liberalización económica y de un patrón de crecimiento económico sostenido. El argumento general es que en India la "biopolítica funciona a través de procedimientos burocráticos 'normales', que terminan des-politizando la muerte de los más pobres" (Gupta, 2012, p. 279). Así plantea que los resultados aparentemente arbitrarios de los programas estatales de 'cuidado de la población' son producidos

3 La expresión Red tape, que al parecer surge en el siglo XIX, rememora el uso de cintas rojas para organizar ciertos documentos en la monarquia española del siglo XVI. 
sistemáticamente por los mismos mecanismos y prácticas dirigidas a disminuir la pobreza extrema. En la trama del libro se imbrican este tipo de enunciados generales de corte teórico con viñetas etnográficas que resultan de más de veinte años de trabajo con funcionarios del nivel medio y bajo en el estado de Uttar Pradesh, norte de India.

Red Tape puede leerse como un recorrido por o una sintesis de todo el trabajo de Gupta, el mismo que lo ha convertido en una referencia fundamental desde Estados Unidos, de un campo conocido como la antropología del estado (Gupta y Sharma, 2006; Palacios, $2015)^{4}$. Como una forma de explorar algunos supuestos de este campo, en lo que resta de esta reseña quiero enfatizar en los aportes y limitaciones de Red Tape para abordar conceptual y metodológicamente al estado, teniendo en mente algunos aportes al estudio del estado desde Colombia.

Un rápido recorrido por los capítulos de Red Tape nos permitirá reconocer algunos momentos y lenguajes dominantes de la antropología del estado. Como su punto de partida es examinar las prácticas cotidianas de los burócratas y las interacciones entre ellos y los ciudadanos -donde formas de cuidado y violencia coexisten-, Gupta organiza el libro en tres grandes partes que dan cuenta de esas prácticas e interacciones: corrupción, escritura y gubernamentalidad. La segunda parte no solo explora cómo las prácticas y las lógicas de la corrupción funcionan en tanto mecanismos que re-producen la violencia estructural, sino cómo determinadas narrativas sobre la corrupción la justifican y legitiman (Cfr. Gupta, 1995). La tercera parte, titulada "Inscripción", cuestiona la idea de que las prácticas de escritura de los burócratas son apenas un mecanismo de dominación de la gente pobre en contextos de analfabetismo extendido. La cuarta parte compara dos programas dirigidos a mujeres pobres para explorar las continuidades y diferencias entre la gubernamentalidad 'clásica' y 'la neoliberal' en India.

4 Dos de sus artículos en particular (Gupta, 1995; Ferguson y Gupta, 2002) son de los textos más consultados y citados en esta literatura (Curtis, Ferguson y Gupta, 2011; Gupta, Nugent y Sreenath, 2015). En Colombia ambos artículos son referencias comunes y el primero de ellos acaba de ser traducido al español (Palacios, 2015). 
La primera parte discute los conceptos de pobreza y violencia estructural. Inspirado en Agamben, Gupta señala que la violencia estructural -que él define como la deliberada y evitable muerte de las personas más pobres- es constitutiva del estado moderno, una idea que complica el reconocido planteamiento foucaultiano del 'dejar morir'. De ahí que adopte una comprensión de la pobreza no como una cualidad en sí misma, sino como una forma de violencia estructural que puede ser explicada. Sin embargo, Gupta afirma que las ideas de Agamben están basadas en una imagen monolítica del estado moderno que no permite explicar la muerte de las personas más pobres en un contexto en el que se ha supuesto su inclusión a la 'democracia formal' y al estado desarrollista. En este punto insiste en que, para superar las visiones monolíticas y normativas sobre la burocracia estatal, es necesario mostrar las prácticas cotidianas que producen tanto 'la arbitrariedad' como 'la indiferencia' ante los impactos de las políticas y programas que reproducen la pobreza extrema (Cfr. Herzfield, 1992).

De esta discusión conceptual se desprenden unas premisas centrales: el estado debería ser estudiado etnográficamente en relación con otros problemas y no como un 'objeto' en sí mismo, y esas relaciones cambiantes deben llevar a una constante teorización de lo que entendemos por estado. Así, Gupta no apuesta por una categoría analítica fija sino que plantea elementos para su comprensión y la de sus burocracias como problemas etnográficos. En mi opinión, es este último el gran aporte del libro, y no tanto lo que deriva de su más reciente interés por explicar la pobreza y la violencia estructural, problemas al final supeditados a su análisis sobre el estado.

A grandes rasgos veo tres fuentes distintas que soportan la comprensión de Gupta sobre el estado. Primero, su lectura de la conferencia de Phillip Abrams (1977/2006), desde la cual entiende que es necesario distinguir analíticamente entre 'el sistema de estado', como el aparato institucional y sus prácticas, y la 'idea del estado': cómo es imaginado concretamente y cómo las ideas sobre él legitiman la dominación política. Aunque el primero es un importante punto de entrada etnográfico, el segundo debe ser un objeto de estudio central para cuestionar la imagen del estado como una entidad dada y una 
realidad ontológica (Gupta, 2012, p. 43). Segundo, Red Tape es un extenso ejemplo de la crítica poscolonial a ciertas concepciones liberales y eurocéntricas (ver Hansen y Stepputat, 2001). En palabras de Gupta, desde 'una perspectiva antiorientalista' cada parte cuestiona ideas universalistas y expansionistas, pero limitadas y provinciales, sobre la corrupción (Gupta, 2012, p. 76, 79, 91), sobre la relación entre alfabetismo y democracia (Gupta, 2012, p. 220-221), y sobre el estado de bienestar y el liberalismo (Gupta, 2012, p. 248, 276). Tercero, y más determinante a mi juicio, trata de reconciliar las premisas fundamentales de Foucault y de los estudios de gubernamentalidad con la etnografia del estado (Curtis, Ferguson y Gupta, 2011). Esto implica distanciarse de algunos de los enunciados del mismo Foucault (1978/2006) que conducian a un abandono del estudio del estado (Cfr. Mitchell, 1999) y al mismo tiempo, a no limitar la gubernamentalidad ni a una sola forma histórica de gobierno ni exclusivamente a la relación entre estados y ONG's (Cfr. Ferguson y Gupta, 2002). Red Tape sin duda sigue los objetos y las categorias provenientes de los estudios de gubernamentalidad (Cfr. Inda, 2005). Aunque Gupta no lo afirma, cada parte del libro enfatiza más en unas dimensiones de estos estudios que en otras (fiel a la propia trayectoria del autor): discursos, narrativas y racionalidades -en corrupción-, prácticas y técnicas -en inscripción-, y técnicas y formación de sujetos -en gubernamentalidad $^{5}$.

Desde este marco Gupta propone una 'mirada desagregada del estado', quizá su apuesta metodológica más significativa. Esta mirada enfatiza el carácter contradictorio, translocal, pluricentrado y multi-nivel del estado, para cuestionar la idea tan naturalizada y problemática de este como una entidad coherente y unificada. De ahí, Gupta afirma que cualquier estudio sobre el estado debe aclarar y está mediado por el nivel, la ubicación institucional y la rama del estado estudiada. Esta mirada debe conducir a una conciencia sobre la parcialidad y la situacionalidad del análisis, y por esta vía, el

Así, la etnografia del estado a lo Red Tape se limita a examinar prácticas, interacciones y discursos, casi siempre en instituciones y actores que son considerados como estatales. Según Gupta, esto es necesario para contrarrestar la ubiquidad del estado que él ve en Gramsci y Althusser. De allí, la diferencia con otros enfoques que no se concentran exclusivamente en instituciones estatales como los de Truillot (2001) o Corrigan y Sayer (1985). 
etnógrafo debe reconocer su lugar en las mismas prácticas e instituciones estatales. Este reconocimiento de la especificidad no niega las conexiones translocales ni ver lo local en tanto atravesado y constituido por otros niveles. Así, Gupta clarifica que su etnografia no es sobre 'el estado local' como una cosa fija y cerrada que se opone al estado regional o nacional, sino sobre el estado 'en' el nivel local -una distinción significativa. De acuerdo con él, esta mirada desagregada es importante en términos políticos ya que permite dar cuenta de las formas de reificación del estado que las élites emplean, resalta sus múltiples tensiones y contradicciones y abre la posibilidad de plantear nuevas tácticas para la acción política y en contra de la aparente arbitrariedad de los programas burocráticos.

Gupta logra desarrollar estos principios en relación con su énfasis usual en discursos e interacciones mundanas -reflejado desde la portada- a diferencia de otros estudios que privilegian coyunturas y eventos puntuales. La tercera parte es particularmente novedosa y brillante al mostrar cómo la elaboración de quejas y reclamos revela tensiones entre distintos funcionarios estatales y algunas estrategias subalternas de resistencia en contextos de restringido alfabetismo pero intensa democracia. Asimismo, explora la reverencia a los documentos de parte de los funcionarios estales, en relación con la construcción de capital cultural y académico. Igualmente, el último capítulo (el séptimo de la cuarta parte) revela de forma detallada las similitudes entre un programa neoliberal (Mahila Samakhya) y un programa clásico del estado de bienestar (el Servicio Integrado de Desarrollo Infantil) en las técnicas de dominio y vigilancia y en la explotación de mujeres subempleadas, y los cambios marcados por las transformaciones de la economía política india y la influencia de los discursos transnacionales de desarrollo de corte neoliberal.

Sin embargo, justo en la cuarta parte y en el epílogo, empiezan a ser más claras las limitaciones para entender el estado y la violencia estructural. El epílogo (una suerte de capítulo corto adicional) explora un paisaje más amplio de proyectos y luchas políticas (alrededor del acceso a la tierra, por ejemplo), la transformación de la economía india hacia los servicios (la cual no permite solucionar el desempleo 
rural) y el rol de las clases industriales emergentes en los programas de desarrollo neoliberal y en la mutación de la hegemonía en India. Así y a pesar de las referencias a Gramsci hechas desde el inicio, en la mayoría del libro es clara la ausencia de un análisis en términos de hegemonía, de relaciones de fuerza y de cambios en la economía politica para responder a sus preguntas iniciales.

Por esta vía, creo que hubiera sido clave un mayor compromiso con el proyecto más amplio de Abrams (1977/2006), para así entender histórica y etnográficamente las luchas entre distintos actores por el dominio y la sujeción política. Esta falta de análisis sobre la relación entre quiénes y cómo, es evidente cuando Gupta usa la palabra estado como sujeto u objeto en muchas frases a lo largo del libro, sin ningún detalle o traducción, y muy a pesar de su interés en no reificar al estado. Cuando uno habla de estado este es uno de los retos más grandes: tratar de mostrar no solo el cómo, sino los quiénes en unas relaciones muy concretas, para no hacer del estado un sujeto dado. Además, creo que estas limitaciones tienen que ver con que Gupta está fuertemente plegado a una etnografia en términos de gubernamentalidad donde, a pesar de todos los esfuerzos, el estado termina siendo un elemento distinguible, un conjunto de instituciones o incluso, un actor más (aunque 'desagregado') de una red de actores y formas de gobierno. También creo que esto se relaciona con una comprensión de lo etnográfico más preocupada por las prácticas, discursos y narraciones, y mucho menos por dar cuenta de posiciones sociales, relaciones y luchas entre distintos actores.

Para finalizar, debo aclarar que estas críticas no solo surgen de los caminos que Gupta abre en las últimas páginas de su libro, si no de pensar sus preguntas iniciales en relación con algunos estudios sobre el estado en Colombia. En general, estos estudios revelan actores, poderes politicos, disputas y relaciones concretas (incluidas las de parentesco), que están en juego cuando hablamos del estado en lo local y lo regional (por nombrar solo dos grandes ejemplos, ver Ocampo, 2014; Ramírez, 2001). Algunos de ellos, inspirados en enfoques más cercanos a Abrams (Corrigan y Sayer, 1985; Joseph y Nugent, 1994/2002, han enfatizado en una concepción de formación 
del estado que nos permitiría entender 'lo fallido' y las supuestas ausencias del estado como una producción misma de las trayectorias de poder, de la comprensión de lo político y de la cristalización de luchas entre distintos actores y proyectos de dominación en contextos especificos (González, Bolivar y Vásquez, 2003; González y Ocampo 2006; Ramírez, 2001). Así, estas miradas permitirian explicar más complejamente la pregunta por lo fallido que le preocupa a Gupta, cuestionando visiones normativas sobre el estado (Ver también la discusión de Buchelly, 2015). Por esta vía, complejizando también 'la mirada desagregada', estos trabajos proponen analizar cómo se configuran "presencias diferenciadas del estado" (González, 2014) y cómo lo marginal y periférico, como lo fallido, son producciones sociales y no realidades dadas (Ramírez, 2001; Serje, 2013).

Claro, estos estudios se ven enfrentados a la dificultad de usar el estado como una categoría analítica, sin caer en su reificación y $\sin$ imponerla como única forma de entender lo político (Bolívar, 2010). También es cierto que estas aproximaciones han sido fruto de una preocupación por explicar el estado en medio de la guerra y la violencia política. Por eso último, aunque hay importantes trabajos sobre la violencia más allá del conflicto armado, las preguntas de Gupta sobre la relación entre violencia estructural y estado son importantes hoy en Colombia. Sin duda, este tipo de preguntas se hacen más urgentes en un contexto donde el desarrollo y el crecimiento económico son constantemente invocados como promesas del posconflicto, mientras la pobreza extrema y la desigualdad, y las muertes que producen, continúan sin que se 'deslegitimen los regimenes dominantes'.

\section{Referencias}

Abrams, P. (1977/2006). Notes on the difficulty of studying the state. En A. Sharma y A. Gupta (Eds.), The anthropology of the state (pp. 112-130). Malden, MA Oxford: Blackwell. 
Bolivar, I. (2010). Formación del Estado y biografia de las categorías. Nómadas, 33, 93- 107.

Buchelly, I. L. F. (2015). Activismo burocrático: La construcción cotidiana del principio de legalidad. Bogotá: Universidad de Los Andes, CIJUS.

Corrigan, P. y D. Sayer. (1985). The great arch: English State formation as cultural revolution. London: Blackwell.

Curtis, J. Ferguson J. y Gupta, A. (2011). Interview: anthropological research on NGOs. En PoLAR. Recuperado de http://www.polaronline.org/spillover/ research-on-ngos/interview-ferguson-gupta/

Ferguson, J. y A. Gupta. (2002). Spatializing States: Toward an ethnography of neoliberal governmentality. American Ethnologist,29(4), 981-1002.

Foucault, M. (1978/2006). Seguridad, territorio, población: Curso en el Collége de France (1977-1978). Buenos Aires: FCE.

González, F. E., Bolivar, I. J. y Vásquez, T. (2003). Violencia política en Colombia: De la nación fragmentada a la construcción del Estado. Bogotá: Centro de investigación y educación popular (CINEP).

González, F. E. y Ocampo, G. I. (2006). Globalización, cultura y poder en Colombia: Una mirada interdisciplinar. Medellin: Universidad de Antioquia.

González, G. F. E. (2014). Poder y violencia en Colombia. Bogotá: Cinep.

Gupta, A. (1995). Blurred boundaries: the discourse of corruption, the culture of politics and the imagined state. American Ethnologist: the Journal of the American Ethnological Society 22, 375-402.

Gupta, A. (2012). Red Tape: Bureaucracy, Structural Violence, and Poverty in India. Durham: Duke University Press. 
Gupta, A. y Sharma, A. (2006). Rethinking Theories of the State in an Age of Globalization. En A. Sharma y A. Gupta (Eds.), The Anthropology of the State (pp.1-42). Malden, MA; Oxford: Blackwell.

Gupta, A., Nugent, D. y Sreenath, S. (2015). State, corruption, postcoloniality: A conversation with Akhil Gupta on the 20th anniversary of "Blurred Boundaries" American Ethnologist, 42(4), 581-591.

Hansen, T. B. y Stepputat, F. (2001). States of imagination: Ethnographic explorations of the postcolonial state. Durham N.C.: Duke University Press.

Herzfeld, M. (1992). The social production of indifference: Exploring the symbolic roots of Western bureaucracy. New York: Berg.

Inda, J. (2005). Analytics of the Modern: an Introduction. En Anthropologies of Modernity: Foucault, Governmentality, and Life Politics (pp. 1-20). Malden, MA: Blackwell Pub.

Joseph, G. M. y Nugent, D. (1994/2002). Aspectos cotidianos de la formación del Estado: La revolución y la negociación del mando en el México moderno. México: Ediciones Era.

Mitchell, T. (1999). Society, Economy and the State Effect. En A. Sharma y A. Gupta (Eds.), The Anthropology of the State (pp. 169-186). Malden, MA; Oxford: Blackwell.

Ocampo, G. I. (2014). Poderes regionales, clientelismo y Estado: Etnografias del poder y la politica en Córdoba, Colombia. Bogotá: Cinep, ODECOFI.

Palacios, M. (2015). Prólogo. En Abrams/Gupta/Mitchell, Antropología del Estado (pp. 9-15). México: FCE.

Ramírez, M. C. (2001). Entre el Estado y la guerrilla: Identidad y ciudadanía en el movimiento de los campesinos cocaleros del Putumayo. Bogotá: Instituto Colombiano de Antropología e Historia, Colciencias. 
Serje, M. (2013). El mito de la ausencia del Estado: la incorporación económica de las "zonas de frontera" en Colombia. Cahiers Des Amériques Latines, 71(3), 95-118.

Sharma, A. y Gupta, A. (2006). The Anthropology of the State. Malden, MA; Oxford: Blackwell.

Trouillot, M. R. (2001). The Anthropology of the State in the Age of Globalization: Close Encounters of the Deceptive Kind. Current Anthropology, 42, 125-138.

\section{Cómo citar esta reseña}

Arias, J. (2016). La antropología del estado desde Akhil Gupta: a propósito de Red Tape: burocracia, violencia estructural y pobreza en India. [Reseña del libro Red Tape: Bureaucracy, Structural Violence, and Poverty in India. por A. Gupta]. Universitas Humanística, 82, 463-473. http://dx.doi.org/10.11144/ Javeriana.uh82.aeag 